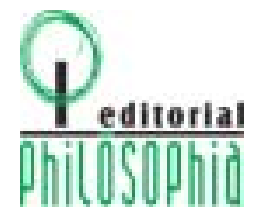

\title{
CONSIDERAÇÕES CRÍTICAS SOBRE A ABORDAGEM QUANTIFICACIONAL DOS DEMONSTRATIVOS - ACERCA DO LIVRO DE JEFFREY KING ${ }^{1}$
}

\author{
André Leclerc \\ Universidade Federal da Paraíba \\ aleclerc@openline.com.br
}

RESU M O : O s demonstrativos foram considerados tradicionalmente como expressões referenciais. É o que encontramos na história da filosofia desde o início da tradição gramatical ocidental que sempre tratou os demonstrativos como pronomes. A maior provocação no livro de King consiste precisamente em romper com essa tradição ao apresentar os demonstrativos complexos ("esta mesa", "aquele homem" etc.) como termos quantificados (e, portanto, termos não-referenciais). King apóia seu tratamento sobre exemplos escolhidos que parecem favorecer sua tese, como "aquele arquiteto que construiu essas pi râmides era um gênio", quando o locutor da frase não tem nenhum alvo referencial em mente e não pode apontar para ninguém no contexto da enunciação. M ostrarei: 1) queo tratamento de King representa uma tremenda ruptura com a tradição e desconsidera as intuições de gerações e gerações de lingüistas e filósofos da linguagem; 2) que King apóia sua tese em evidências de natureza puramente sintática sem discutir metodologicamente o peso que devemos atribuir a essas evidências sintáticas nas discussões de semântica; 3) que King não leva em conta todos os dados disponíveis, forçan do os dados por ele recolhidos a entrar numa mesma teoria unificada, mas, para isso, paga um preço alto: uma redundância inaceitável na semântica das expressões demonstrativas si mples.

Pal avras-chave: Demonstrativos, referência direta, quantificação, sintaxe/ semântica.

INTRODUÇÃO

No ano passado, o MIT lançou o segundo livro da série Contemporary Philosophical Monographs, intitulado Complex demonstratives: a quantificational approach, da autoria de Jeffrey $C$. King. 0 tratamento dos demonstrativos e de outras formas de dependência contextual estiveram no centro de muitas discussões, nas últimas décadas, em filosofia da linguagem, particularmente na teoria da referência, bem como em filosofia da mente, com a 
emergência do externalismo. N esse contexto, o que pensar de uma teoria que pretende que os demonstrativos não sejam termos referenciais? A pós um resumo da teoria de King, apresentarei, contra ela, as seguintes considerações críticas: 1) devemos reconhecer que há aqui uma tremenda ruptura com a tradição - para 0 ckham, Arnauld de Port-Royal e, mais recentemente, Frege, Russell, Reichenbach, Bar-H illel, Montague, Kaplan, Evans, Perry, Wettstein e outros, os demonstrativos são termos singulares referenciais - , e a formação de um juízo considerado sobre uma teoria que nega que os demonstrativos sejam termos referenciais não pode desconsiderar a intuição desses filósofos; 2) King apóia-se em evidências sintáticas, construindo assim uma analogia entre 0 comportamento sintático dos demonstrativos com outros quantificadores, sem discutir o peso que temos de atribuir à evidência sintática nas discussões de semântica; 3) outro tipo de evidência não foi levado em conta por King: a maioria das línguas comporta uma distinção dentro da categoria dos demonstrativos que se apóia numa distinção subjacente entre referente próximo (C eci, This, I sto etc.) e referente distante (C ela, That, Isso etc.) - a abordagem de King privilegia 0 that em detrimento de this e focaliza demais os NDNS uses (no demonstration no speaker's reference) dos demonstrativos; 4) indaga-se finalmente: será que essa teoria é realmente semântica como pretende o autor, que se apóia enfaticamente nas intenções dos locutores (que não são marcadas nas frases) e numa teoria da elipse que não tem o aval de muitos lingüistas? Essa teoria da elipse funciona muito mal para certos exemplos envolvendo "demonstrativos simples" como "Isto é uma árvore", "Isto é uma vaca" etc. $\mathrm{H}$ averia nesses casos elipse de um predicado (sortal). King defende sua teoria pretendendo que ela abarque mais dados lingüísticos e que, portanto, ela seja mais abrangente do que as concepções rivais desenvolvidas no âmbito da teoria da referência direta. Contestarei essa defesa de King, mostrando justamente que ele não leva em conta todos os usos. Mostrarei também que o tratamento de King dos usos N D N S e dos demonstrativos simples é 
altamente implausível, pois envolve uma redundância inadmissível no sentido (convencional) dessas expressões, se aceitarmos simplesmente a idéia de que a linguagem que falamos hoje (i.e., o conjunto das convenções lingüísticas de uma determinada língua num determinado período) éfruto de séculos de evolução, resultado de milhares de decisões racionais tomadas por gerações de fal antes. Se a razão tem al go a ver com a linguagem e com sua evolução, a tendência normal é a eliminação, e não a multiplicação, das redundâncias.

\section{A teORIA DE KING: UMA RUPTURA VIOLENTA COM O PASSADO}

A filosofia da linguagem e da mente das últimas décadas prestaram muita atenção ao tratamento das expressões demonstrativas, particularmente desde o famoso artigo de Y. Bar-Hillel, em 1954, "Indexical expressions", ${ }^{2}$ que sugeriu a idéia de que a pragmática teria como objeto específico os indexicais e demonstrativos. Logo depois M ontague retomou essa idéia. Sabemos hojeque, na verdade, o que M ontague fezera uma extensão da model theoretical semantics, e os tratamentos mais recentes e muito influentes de Evans, Kaplan, Wettstein e Perry pertencem claramente à semântica. Não é um exagero dizer que 0 assunto mais importante da segunda metade do século XX em filosofia da linguagem foi o estudo da dependência contextual de nossas enunciações e de nossos pensamentos. Pois, antes de Bar-Hillel, poucos autores de destaque trataram dos demonstrativos e indexicais, e quando eles se deram essa pena, foi na maioria dos casos só en passant e de maneira bastante programática. Assim, Frege, em "Der Gedanke", de 1918, tentou determinar que tipo de pensamento expressava a frase "Eu fui ferido" proferida pelo Dr. Lauben. Em 1940, Russell, em A n inquiry into meaning and truth, introduziu a noção de "particulares egocêntricos" e manteve a idéia de que os demonstrativos eram como nomes próprios (lógicos) que referiam sem descrever. Em 1947, Reichenbach, nos seus Elements of symbolic logic, reforçou, 
por sua vez, a característica de reflexividade dos demonstrativos e indexicais com a noção de token-reflexives. Em 1949, Arthur Burks ("Icon, index and symbol"), menos conhecido, deu também à semântica dos indexicais e demonstrativos uma contribuição importante, justamente relembrada por Perry num texto recente intitulado "Indexicals and demonstratives", de 1997. ${ }^{3}$ Todos esses autores tratam os demonstrativos como termos referenciais. A Iguns (Kaplan, Perry, Castañeda e W ettstein) seguem R ussell e dizem que são diretamente referenciais; as frases declarativas contendo demonstrativos expressam proposições singulares, proposições que contêm pelo menos um indivíduo como constituinte. Entreautores mais antigos, 0 ckham, na segunda parte de sua famosa Summa totius logicae, trata dessas proposições básicas que têm de ser verdadeiras para que outras proposições tenham um valor de verdade (o que chamamos desde Strawson de "pressuposições semânticas"), e essas proposições, pelo menos aquelas do tipo mais básico, são da forma "Isto éF" (T hisisF ). Sem dúvida: para O ckham, os demonstrativos são termos referenciais, e provavelmente termos diretamente referenciais, mesmo que ele nunca tenha explicitado essa idéia. ${ }^{4}$ Arnauld de Port-Royal, junto com N icole, elaborou, na quinta edição de 1683 da Logique ou l'A rt de penser, uma teoria ideacional interessante dos demonstrativos que ser ve para expressar idéias "confusas" representando objetos presentes no contexto de enunciação. D e acordo com a tradição gramatical, A rnauld e N icole tratam os demonstrativos como pronomes, que certamente entram na categoria dos termos singulares e referenciais.

Portanto, a meu ver, a principal audácia de Jeffrey C. King é justamente a de negar que os demonstrativos sejam termos referenciais. $^{5} 0$ que ele chama de that phrases, para designar os demonstrativos complexos, têm mais a ver, segundo ele, com os quantificadores. Parece que temos aqui uma espécie de tentativa de repetir a façanha de Russell, de tratar as descrições definidas como sincategóremas, enquanto outros, como Frege, os consideravam como termos singulares referenciais, como Strawson décadas depois. 
0 artigo definido é um quantificador derivado, ao qual podemos atribuir uma "definição contextual". Na análise de Russell, a contribuição proposicional das descrições definidas é de natureza geral ou conceitual. Por analogia, na teoria de King, as that phrases, como "este homem", "aquele animal", "esse carro" etc., dão uma contribuição proposicional bem diferente da dos nomes próprios: ao invés de introduzir um indivíduo no discurso, os demonstrativos, segundo King, em virtude de seu significado lexical, introduzem propriedades. 0 significado lexical (fora de qualquer contexto) de "esse" (that) seria uma relação quaternária: " ___ e ___ são unicamente _ num objeto e ele é___", com duas propriedades (preenchendo o segundo e terceiro lugares) determinadas pelas intenções do locutor, as quais podem ser perceptuais ou descritivas. U ma frase da forma "Este $F$ é $G$ " expressaria, quando a intenção do locutor é perceptual, por exemplo, uma proposição com os seguintes constituintes: "a propriedade de ser $\mathrm{F}$ e a propriedade de ser idêntico a b (b sendo o objeto da percepção) têm a propriedade de ser unicamente instanciadas juntamente em w et num objeto e ele tem a propriedade G" (voltaremos a esse assunto daqui a pouco). Ao invés de encarar os demonstrativos como pronomes, King os trata sintaticamente como determinantes (determiners) junto com os outros quantificadores (todos, um, a maioria, alguns etc.). Ao invés de apoiar-se numa analogia com os indexicais que manifestam as mesmas características de ref lexividade e depen dência contextual que os demonstrativos, King constrói seu tratamento teórico a partir de uma outra analogia, a dos demonstrativos com os quantificadores. Para ter sucesso nisso, ele escolhe bem seus exemplos e apóia-se em evidências de natureza sintática. Tentará depois esten der seu tratamento dos demonstrativos complexos a outros usos dos demonstrativos, como "este é $F$ " (demonstrativos simples).

D oravante, salvo indicação contrária, todos os exemplos usados são da autoria de King. Devo observar que as minhas traduções desses exemplos às vezes en volvem outros demonstrativos, além dos habituais "isto" e "isso", como "aquele", por exemplo, pois 
o português, bem como o francês, é muito mais rico em demonstrativos do que 0 inglês.

A estratégia argumentativa de King consiste em selecionar e privilegiar certos usos que se encaixam muito mal na teoria da referência direta. Por isso o primeiro capítulo é intitulado "A gainst direct reference accounts" e privilegia exemplos que ele designa como usos N D NS. Esses usos de that phrases não envolvem nenhuma demonstração (gesto de apontar para algo no ambiente físico imediato) por parte do locutor, e ele não precisa também ter nenhum alvo referencial em mente. Assim, posso dizer, adotando um tom meio admirativo: "Esse hominídeo que descobriu como acender fogos era um gênio" (T hat hominid who discovered how to start fires was a genius). Com base nos usos atributivos das descrições definidas à la D onnellan, poderíamos parafrasear: "A quele hominídeo, não importa quem, que descobriu como acender fogos era um gênio". Fica claro quenenhum indivíduo foi introduzido na proposição como um dos seus constituintes. É notável que, logo no início de seu livro, King adote uma concepção das proposições como entidades estruturadas cujos constituintes são indivíduos, propriedades e relações - algo que ele tem em comum com muitos partidários da teoria da referência direta. Ele aceita, portanto, a idéia de proposição singular ou russelliana. Sua oposição à teoria da referência direta para os demonstrativos não depende, como é 0 caso dos fregeanos ou neofregeanos, de uma rejeição incondicional das proposições singulares. Mas, ao invés de alinhar os demonstrativos aos nomes próprios e aos in dexicais, King escolhe a direção oposta. Portanto, para ele, frases conten do demonstrativos servem para expressar proposições cujos constituintes são todos de natureza geral, i.e., propriedades.

Já mencionei que os demonstrativos, na teoria de King, têm como significado lexical uma relação quaternária; os segundo e terceiro lugares daquela relação são saturados por propriedades fornecidas e determinadas pelas intenções do locutor. Às vezes a intenção é "perceptual" e, com isso, King entende a intenção de 
"falar sobre" um objeto percebido pelo locutor no contexto de enunciação. N esse caso, a propriedade que preenche o segundo lugar é, como já vimos, do tipo "ser idêntico a b" (onde b é o objeto percebido). 0 bviamente, essa propriedade não pode ser instanciada por mais de um objeto em qualquer mundo possível onde b exista, daí o caráter de rigidez associado, às vezes, aos demonstrativos. Seja a frase "Este pássaro é um sabiá" (exemplo meu), pronunciada por mim no momento t. Essa frase expressaria a seguinte proposição: a propriedade de ter uma única instância comum em w e t da propriedade de ser um pássaro e da propriedade de ser idêntico a $\omega$ e $\omega$ tem a propriedade de ser um sabiá. (A qui $\omega$ representa o objeto percebido.) 0 pássaro que estou percebendo em w e t tem a propriedade (que é um propriedade de segunda ordem - uma propriedade de um par de propriedades) de ser a única instância comum da propriedade de ser um pássaro e da propriedade de ser idêntico ao objeto que estou percebendo, e este tem a propriedade de ser um sabiá. É a propriedade de ser idêntico a $\omega$ que explica aqui a rigidez: qualquer que seja a situação contrafactual, nenhum outro objeto pode instanciar aquela propriedade, a não ser o objeto efetivamente percebido por mim em w et. A intuição semântica de rigidez associada ao uso dos demonstrativos e capturada por Kaplan com a idéia de proposição singular é aqui explicada pela instanciação da propriedade relacional e essencial de ser idêntico a si mesmo (ao objeto percebido), sem precisar da referência direta. A propriedade de ser idêntico ao objeto percebido, a qual é associada por King aos usos dos demonstrativos que manifestam a propriedade semântica de rigidez, é tal que, no uso de uma frase da forma "este $F$ é $G$ ", a inten ção perceptual do locutor determina (picks out) o mesmo objeto em todos os mundos onde este existe, não importando se este tem ou não a propriedade $F$.

Em outras circunstâncias, a propriedade fornecida pela intenção do locutor é descritiva, e aí não tem a rigidez associada a esses usos. É o caso do hominídeo genial que descobriu a arte de acender fogos, e em geral dos usos N DN S examinados por King. A meu ver, 
o tratamento de King, nesses casos, é muito deselegante por causa da redundância que ele introduz para promover um tratamento unívoco dos demonstrativos, juntando os usos que manifestam rigidez com os outros que envolvem uma intenção descritiva do locutor. N outras palavras, King defende que as that phrases não são ambíguas e que nós não devemos dividir a categoria em duas partes, uma associada à teoria da referência direta, e a outra à abordagem quantificacional. Veremos como King trata o exemplo em questão. A frase "Esse hominídeo que descobriu como acender fogos era um gênio" expressaria a seguinte proposição (notem a redundância): a propriedade de ser um hominídeo que descobriu como acender fogos e a propriedade de ser um hominídeo que descobriu como acender fogos são unicamente instanciadas em comum num objeto $x$ ex era um gênio. A verdade dessa proposição numa circunstância de avaliação qualquer $C$ não requer que o mesmo indivíduo que possui essa propriedade em w e t (o contexto de enunciação atual) esteja envolvido em C. É suficiente, para a verdade da proposição, quehaja um indivíduo em $\mathrm{C}$ satisfazendo a propriedade determinada pela intenção do locutor.

King fornece, para defender sua teoria, certos usos de that phrases que ele chama de usos Q I (por quantifying in). Aí a evidência fornecida é de caráter sintático. Seja a frase "todo pai receia aquele momento quando seu filho mais velho vai embora de casa" (Every father dreads that moment when his oldest child leaves home). A qui, "aquele momento" não é referencial. Temos com este exemplo e outros do gênero o caso de um quantificador ligando uma variável (na forma de um pronome) aparecendo no escopo de um outro. $\{\forall x[$ (x é um pai) $\rightarrow \exists$ y (y é o momento de ver o filho de $x$ ir embora da casa) $\wedge$ $x$ tem medo de $y$ ]\}. 0 comportamento sintático do demonstrativo nesse tipo de uso não parece diferente do comportamento de outros quantificadores em frases como: "C ada homem ama al guma mulher que ele já beijou", ou "Cada mulher ama o homem que ela beijou pela primeira vez". King examina cuidadosamente o comportamento sintático dos demonstrativos em vários outros contextos que 
envolvem a negação, modalidades everbos de atitudes. Não entrarei nesses detalhes de sua teoria porque isso levaria muito tempo.

\section{CONSIDERAÇÕES CRÍTICAS}

(1) Vimosque King, com sua tese de que os demonstrativos não são termos singulares referenciais, rema contra a corrente e rompe com séculos de semântica filosófica. Isso não constitui um argumento muito forte contra a teoria quantificacional, mas ajuda na hora de atribuir o fardo da prova. Qual é a maior motivação da teoria quantificacional? Além da evidência sintática que consideraremos daqui a pouco, fica claro que é aparentemente a convicção de que as that phrases constituem uma categoria bem unificada e devem ser, portanto, tratadas univocamente. Vimos que certos usos de demonstrativos envolvem a propriedade semântica de rigidez, e outros não. U ma tal diferença é notável. Então, qual é, segundo King, a característica unificadora dos demonstrativos? Sua resposta vem no final do livro: é que as that phrases necessitam de uma saturação por propriedades determinadas pelas inten ções (perceptuais ou descritivas) do locutor. Essa característica unificaria a categoria. U ma outra motivação vem do comportamento sintático exibido pelas that phrases. Tem enfim o argumento metodológico: um tratamento unificado que leva em conta um maior número de dados deve ser preferido a qual quer outro tratamento que favorece a tese da ambigüidade.

O que cria confusão aqui, talvez, é a pal avra "uso". Podemos fazer das palavras o uso mais variado, como Wittgenstein nos ensinou. Mas ficaria ridículo exibir orgulho de uma teoria unificando os usos, digamos, da palavra "fogo", uma vez usada na frente do pelotão de execução, outra vez na frente de um incêndio irrompendo; no primeiro caso com a intenção de matar, e no segundo com a intenção de avisar do perigo. É a mesma palavra, mas as intenções não são as mesmas! A linguagem é uma instituição social, e o conhecimento semântico é o conhecimento das convenções 
que determinam sistematicamente o significado das palavras e 0 significado das expressões complexas a partir do significado das palavras. A palavra "fogo" é a mesma, mas pelo resto, para fixar em contexto o conteúdo, "o que foi dito", temos de apelar para nossas habilidades hermenêuticas e utilizar todas as indicações contextuais disponíveis. As intenções do locutor são importantes, mas a teoria intencionalista dos demonstrativos e indexicais já foi criticada adequadamente por Wettstein, e seus limites são bem conhecidos. ${ }^{6}$

King, como vimos, aceita a idéia de proposição singular, como os partidários da referência direta, mas não quando se trata dos demonstrativos. Ele concorda também com eles quanto à seguinte conseqüência: um locutor que afirma e pensa (apontando na direção de uma árvore) "este pássaro éum sabiá" não faz nenhuma afirmação e não tem nenhuma crença correspondente se não tiver um pássaro na árvore. Falta um constituinte na proposição, e, portanto, não tem nenhuma proposição. Assim, a crença correspondente não existe, pois uma crença sem conteúdo não é crença nenhuma. Alguns partidários da teoria da referência direta aceitam essa conseqüência, e King concorda com eles quanto a isso. Ele acha que essa intuição é correta. Na sua teoria, ela dá conta disso da seguinte maneira: quando o locutor pensa que percebe um objeto $\omega$ e tem a intenção perceptual de falar sobre $\omega$, masnão tem nenhum $\omega$ presente (a percepção não foi verídica), a proposição expressa tem um buraco (a gap), o que significa que ela não pode ser avaliada semanticamente, o que quer dizer, finalmente, que não há proposição nenhuma. A qui King parece reconhecer que existe al go como uma identificação demonstrativa, mas esta requer normalmente a própria existência do objeto. Q uando o objeto não existe, a identificação não pode ser demonstrativa, mas sim descritiva, e aí a proposição não pode ser singular. Como os pragmatistas nos ensinaram, agimos constantemente com base em nossas crenças. Suponhamos que o locutor do exemplo precedente levanta para pegar seus binóculos a fim de ter uma visão melhor de seu pássaro preferido. Ele então constata que não há pássaro na árvore. Se a 
intuição de King for certa, o locutor em questão deveria chegar à conclusão de que sua ação (levantar e pegar os binóculos) era totalmente irracional e inexplicável. A cho absurdo esse resultado. M inha posição é a de que se a identificação não pode ser demonstrativa porque o objeto não existe, a melhor coisa a fazer é generalizar a proposição e atribuir uma crença falsa, e não uma crença "aparente" ou nenhuma crença. Isso parece a melhor maneira de preservar a racionalidade do agente numa situação dessas. ${ }^{7}$ Precisamos novamente apelar para nossas habilidades hermenêuticas e para todas as indicações contextuais pertinentes, e não só para as intenções do locutor.

(2) Q ual o peso da evidência sintática em semântica filosófica? As evidências reunidas por King a favor de sua tese são bastante impressionantes, masqual o peso disso? 0 próprio King não discute isso. $\mathrm{N}$ a história das ciências da linguagem, James Burnett (Lorde M onboddo), no final do século XVIII, divide as categorias sintáticas em dois grupos, de acordo com a divisão platônica em 0 noma e Rhema; nessa divisão, as preposições, por exemplo, caem do lado dos "verbos" (rhema), e aí temos verbos sem declinação e que não se conjugam. ${ }^{8}$ D ois séculos depois, D onald D avidson, em "The logical form of action sentences", de 1967, faz a mesma coisa e analisa a preposição to na frase "I flew my spaceship to the morning star", como um verbo cujo conteúdo seria algo como "provindo de eindo para". C astañeda, que foi convidado a comentar o texto, estranhou essa análise de Davidson, mas este, na sua réplica, defendeu e manteve seu juízo. M ontague considerava a sintaxe só como uma introdução para a semântica. A mesma frase pode ser decomposta de várias maneiras, mas nem todas são pertinentes para a semântica (quer dizer, para compor os significados). Final mente, se a evidência sintática for tão pesada, deveríamos censurar severamente o discurso dasúltimas décadas sobre os indexicais, particularmente os advérbios de tempo elugar (agora, aqui, ontem etc.) que sempreforam tratados como termos referenciais. A categoria sintática dos advérbios, 
justamente, é bem distinta da dos termos que contêm expressões, como nomes próprios, pronomes etc.; mas isso nunca impediu os filósofos semanticistas de tratar "aqui", "agora", "amanhã" etc. como expressões referenciais...

(3) King, estranhamente, trata sempre do that, e nunca do this. Como já mencionei no início, existe uma distinção distante-próximo subjacente que explica a existência dos pares this/ that, ceci/ cela, isto/ isso, em inglês, francês, português etc. No meio de todos os dados empíricos (sintáticos) relativos aos demonstrativos, King se esqueceu disso! Ele focaliza exclusivamente as that phrases. Minha suspeita éa de que dificilmente se pode encontrar um uso NDNS envolvendo this. (É só substituir o that pelo thisem "That hominid who discovered how to start fires was a genius" para sentir o problema). Pareceme muito mais "natural" tratar o this como (diretamente) referencial. Isto, a meu ver, depõe contra a pretensão de King de oferecer um tratamento unificado das that phrases.

(4) King busca apoiar-se inten samente sobre as intenções do locutor que determinam certas propriedades que se apresentam como constituintes da proposição correspondente. Recorre também à estratégia da elipse para explicar os usos "simples" dos demonstrativos, da forma "isso éF" (That is F), de modo a fazêlos entrar no modelo da relação quaternária que apresentei anteriormente. N esses casos, diz King, há sempre um predicado (um sortal) implícito, não marcado na frase. Mas aí de novo, em nome de uma pseudoregularidade teórica, o espectro feio da redundância volta para assombrar o Rei... U ma frase da forma "isso é F" expressaria uma proposição com os seguintes constituintes: a propriedade de ser idêntico a w e a propriedade de ser idêntico a w são unicamente instanciadas em comum em w et, e ele tem a propriedade de ser $F$. Quando eu digo "isso é uma vaca", apontando para uma vaca que todo mundo vê, em virtude das convenções e regras da língua, a frase expressaria a proposição de que há uma única instância comum em w et da propriedade de ser idêntico a w (ao objeto percebido) e 
da propriedade de ser idêntico a $w$, e ele tem a propriedade de ser uma vaca. 0 preço a pagar para a simetria me parece alto demais. Há muitas coisas implícitas na comunicação, sabemos disso. M as duvido que uma tal redundância seja prescrita pelas regras e conven ções da língua; e se for o caso, o tempo a eliminaria. Ainda que uma certa dose de redundância seja necessária na comunicação humana, a posologia recomendada por King écavalar, desnecessária e deselegante. Sob o pretexto de oferecer uma teoria unificada dos demonstrativos, ele "forçou a barra". 0 livro dele é interessante, provocador e rico. $M$ as quanto a mim, el e não me convence de que sua teoria é preferível à teoria da referência direta, ou que um tratamento unívoco é preferível a um tratamento que se apóia muito mais nas habilidades hermenêuticas dos falantes e aceita como normal o fato que as mesmas palavras possam ser usadas em vários contextos de modo a dar contribuições completamente distintas das condições de satisfação dos enunciados em que ocorrem.

Finalmente, como vimos logo de início, desde 0 ckham pelo menos, as intuições da esmagadora maioria dos filósofos da linguagem convergem: os demonstrativos são termos referenciais. E como dizia Bertrand Russell, com seu humor bem britânico e uma pincelada de ironia: "quando todos os peritos concordam em um ponto, você nunca pode ter absoluta certeza que eles estão totalmente errados".

\section{Agradecimentos}

Ao programa de pós-graduação em Filosofia da UnB e, particularmente, ao Prof. Dr. Nelson Gomes, pelo convite para tomar parte no II Colóquio de Pós-G raduação em Filosofia, na U niversidade de Braślia, ocasião em que este texto foi apresentado.

ABSTRACT: D emonstratives have always been considered as referential expressions. This is what we find out in the history of philosophy since the dawn of our grammatical tradition that always treated demonstratives as pronouns. The most provocative ten et in King's book is precisely that according to which complex demonstratives (like "That 
man", "That table" etc.) are not referential terms but quantified terms. King grounds his treatment on some examples well chosen that seem to favor his theory, examples like: "That hominid that discovered how to start fire was a genius", when the speaker has no referential target in mind and cannot point to someone in the context of utterance. I'll try to show: 1) that King's treatment represents a serious neglect of a long tradition and a disregard of the intuitions of generations and generations of linguists and philosophers of language; 2) that King grounds his thesis on data and evidence of a purely syntactic kind without discussing, methodologically, the weight we should confer to that type of evidence in semantic debates; 3) that King does not take into account all the data available, and that by forcing his data to fit in a unified framework, he has to pay a very high price: an unacceptable redundancy in the semantics of simple demonstratives.

Key words: D emonstratives, direct reference, quantification, syntax/ semantics.

\section{Notas}

1. Apresentado no II Colóquio da Pós-G raduação em Filosofia/ U nB, em agosto de 2002.

2. Bar-H ILlel, Y. Indexical expressions, M ind, v. 63, p. 359-379, 1954.

3. Hale, B.; Wright, C. (O rgs.). A companion to the philosophy of Language. London: Blackwell, 1997.

4. Devo essa informação ao Prof. Claude Panaccio, renomado especialista de 0 ckham.

5. Ver Complex demonstratives: a quantificational approach, M IT Press, A Bradford Book, 2001. (Col. Contemporary Philosophical M onographs), por exemplo, p. 187, nota 36.

6. Ver Wettstein, H. How to bridge the gap between meaning and reference, Synthese, v. 58, p. 63-84, 1984, reimpresso em D avis, Steven (O rg.). Pragmatics. A R eader. O xford: OU P, 1991. p. 160-173.

7. Ver meu "Fallibilism, demonstrative thoughts and russellian propositions", a ser publicado em Principia: RevistaI nternacional de Epistemologia.

8. Ver BuRnETT, J. $O f$ the origin and progress of language, Edinburgh: Menston, Scolar Press, 1967, v. 1 [1773] e v. 2 [1774]. 


\section{R eferências}

Arnauld, A.; Nicole, P. [1662/ 1683]. La logique ou l'art de penser. Paris: Flammarion, 1970.

BAR-H ILLEL, Y. Indexical expressions. M ind, v. 63, p. 359-379, 1954.

Burks, A. Icon, index and symbol. Philosophy and Phenomenological Research, v. IX, p. 673-689, 1949.

Burnett, J. [1773/1774]. Of the origin and progress of language. Edimburgo, v. 1, [1773] e v. 2 [1774]. M enston: Scolar Press, 1967.

DAVIDSON, D. The logical form of action sentences. In: RESHER, N. (O rg.). The logic of decision and action. Pittsburgh: U niversity of Pittsburgh Press, 1967.

Evans, G. Understanding demonstratives. In: Parret, H.; Bo uveresSE, J. (O rgs.). M eaning and understanding. Berlim, N ew York: de G ruyter, 1981. p. 280-303.

Frege, G. [1918]. The Thought. In: P. F. Strawson (O rg.). Philosophical Logic. Oxford: OU P, 1967.

Kaplan, D. Demonstratives. In: Almog, J.; Perry, J.; W ettstein, H. (O rgs.). Themes from K aplan. O xford: O U P, 1989.

KING, J. Complex demonstratives. A quantificational approach. Cambridge (M A): M IT Press, 2001.

Montague, R. Formal Philosophy: selected papers of Richard M ontague. R. Thomason (O rg.). N ew H aven: Yale U niversity Press, 1974.

O cкнам, W. 0 ckham's theory of terms. Primeira Parte da Summa logicae. Tradução de M . Loux. N otre D ame(Ind.): U niversity of N otre D ame Press, 1974.

Perry, J. Indexicals and demonstratives. In: Hale, B.; W Right, C. (O rgs.). A companion to the philosophy of language. London: Blackwell, 1997. 
. The problem of the essential indexical and other essays. Oxford: OUP, 1993.

Reichenbach, H. Elements of symbolic logic. New York: Free Press, 1947.

RusSELL, B. An inquiry into meaning and truth. Middlesex: H armondsworth, 1940.

WetTSTEIn, H. [1984]. How to bridge the gap between meaning and reference. Synthese, v. 58 [1984], p. 63-84; reimpresso em Steven D avis (O rg.). Pragmatics. A Reader. O xford: OU P, 1991, p. 160-173. 